\title{
Concept of Integrating Real Time Field Images in Computer in Process Industries by Removing Separate TVs
}

\author{
A.Justin Diraviam \\ Asst.Professor/CSE \\ Sardar Raja College of Engg \\ Alangulam, Tirunelveli, \\ Tamilnadu
}

\author{
S.P.Victor \\ Research Director/CS \\ St.Xavier,s College \\ Palayamkottai, \\ Tamilnadu
}

\author{
K.Rajappan \\ Principal \\ Sri Subramanya College of \\ Engg \& Tech \\ Palani,Tamilnadu
}

\begin{abstract}
Process Industries are hazardous in nature. They require good instrumentation support for control and monitoring various parameters like pressure, flow, level, temperature, speed, vibration, displacement, etc. Sensors are to be installed at remote fields and measured variables are brought to the operator station for further processing. Initially separate TVs are installed at operator station to view the remote field images (Photographs) taken through analog (conventional) cameras .By viewing both the computer monitor and separate TVs, control signals are generated for abnormalities in the plant by human intervention, which may lead to errors due to human fatigue, sleeplessness in the night, etc .In the present days the signals from sensors are compared and control action is initiated. This also is not full proof method due to errors in sensors. Now due to the invent of digital cameras, analog cameras are replaced by digital cameras at remote fields and the digital signals of real time field images can be directly integrated in to the computer. With the help of emerging DIP(Digital Image Processing)technology these digital images are compared with already stored standard images of remote field and control action is initiated by computer automatically for abnormalities for parameters like level, flow, speed etc by matching the images. Reliability is very high compared to previous methods. . This system gives real time display of various field images super imposing on the animated mimic diagrams in computer monitor, which is not possible in the old environment with analog sensors. MATLAB is extensively used. JPEG image files are used.
\end{abstract}

\section{Keywords}

Sensors, DIP, MATLAB, JPEG

\section{INTRODUCTION}

Most industrial plants are hazardous in nature. The technical development in Electronics, Information technology, Image processing and industrial computers resulted in the development of remote control and monitoring the plants. Remote operation field needs quite a good amount of instrumentation support for control as well as monitoring purposes. Nowadays plant size has grown bigger which needs control of many parameters like flow, level, pressure, speed, temperature, displacement, vibration etc.,

SCADA (Supervisory control and data Acquisition system) or DCS (Distributed Control System)[3] with real time operating system are installed in Process industries based upon geographic distribution of the plant instrumentation. The signals from sensors have to undergo signal conditioning, A/D conversion, engineering unit conversion using linear interpolation method, and limit checking before generating report/control signal for corrective action. The corrective action is done by generating D/A conversion signal from the SCADA/DCS for actuating the control elements.

Sensors like RTD, Thermocouple, strain gauges, tachometers, LVDT's etc generate analog signals. Errors in these sensors are prone to happen which are discussed below. Hence getting accurate results are not possible. . So many correcting methods are to be employed. In the earlier days, when the plant size was smaller, remote field images are brought to the operator station to display through separate TVs. This is because the cameras used were conventional analog cameras which cannot be linked to Real time computers, since computer operate only with digital signals. This TV images give only information about the plants, to view by the operators and site engineers but could not be used for any effective purpose. Control action is to be initiated manually. Nowadays analog sensors are used to bring measured variables of different parameters to the control station. These signals are compared with normal values stored inside computer and control signals are generated by the system during abnormalities. This method is not that reliable due to analog signal transmission errors, sensor frequent calibration requirement, hysteresis condition in sensors, etc.

A new technique to solve these problems is to use Digital Cameras in place of analog sensors. The digital images are taken and continuously transmitted to the operator station. Digital image processing [1] paves way for generating control signals by comparing the just received image with the standard image already stored inside the computer.

\subsection{Objective}

Earlier remote monitoring involves the installation of dedicated hardware links between field camera and TV monitor. For each control operation, the operator has to select the view manually and monitor both operator station terminal and TV monitor. For visualization of the field site the operator relies on manual switching unit. The operator often makes mistakes in selecting the right option. In order to have reliable selection of field image, control and monitoring is integrated along with real time field image processing. This solves switching problem. Automatic control signal generated is based on field image compared with the standard image. 


\subsection{Methodology}

Image integration is a complex video switching system [14] with the objective of maintaining a field target object. In order to perform image integration with computer many tasks are required of the system namely,

- The image must be digitally acquired from the imaging sources, usually a color video camera [7] by sampling procedure to form pixels.

- The Pixels should be continuously logged in to the capturing system.

- Instantaneous field images are transmitted in the network.

- Based on the control operation appropriate field image [1] must selected and displayed in the operator station.

- Displayed image can be analyzed (compared with standard image) by an appropriate algorithm to generate control signal.

\subsection{Advantage of New technique over analog Signals (Sensor)}

Old methods use sensors like RTDs, Thermocouples, Strain gauges, tachometers, LVDTs etc for measuring temperature, pressure, speed, displacement etc. These sensors generate analog signals. Errors in analog signal are highlighted in detail.

The new technique uses Digital Cameras in place of analog sensors. Digital Cameras generate Digital Signal images. The errors highlighted with analog signals are not available with digital signals. Hence the new technique of using digital cameras is superior

\subsection{Errors in Reading Analog Sensors}

No measurement can be made with perfect accuracy but it is important to find out what accuracy actually is and how different errors have entered into measurement. A study of errors is a first step in findings ways to reduce them. Errors may rise from different sources and are usually classified as under.

- Gross error

- Systematic error

- Random error

\subsubsection{Gross Errors}

This class of errors mainly covers human mistakes in reading instruments and recording and calculating measurement results. The experimenter may grossly misread the scale. Although complete elimination of gross errors is probably impossible, one should try to anticipate and correct them. Some gross errors are easily detected while others may be difficult to detect.

\subsubsection{Systematic Errors}

These types of errors are divided into the following:

- Instrumental Errors

- Environmental Errors

- Observational Errors

Instrumental errors arise due to the following main reasons:

- Due to inherent shortcomings in the instrument

- Due to misuse of the instruments

- Due to loading effects of instruments.

Environmental errors are due to conditions external to the measuring device including conditions in the area surrounding the instruments. These may be effects of temperature, pressure, humidity, dust, vibrations or of external magnetic or electrostatic field.
There are many sources of observational errors. The pointer of an instrument rests slightly above the surface of the scale. Thus an error on account of parallax will be incurred unless the line of the vision of the observer is exactly above the pointer. To minimize the parallax errors, highly accurate instruments are provided with mirrored scales.

Systematic errors can also be divided into static and dynamic errors. Static errors are caused by the limitations of the measuring device or the assumption in the physical laws governing its behavior. Dynamic errors are caused by the instruments slow response in following the changes in the measured variable.

Systematic errors have definite magnitude and direction. These are usually more troublesome as repeated measurements may not reveal them.

\subsubsection{Random Errors}

It has been consistently found that experimental results show variation from one reading to another even after all systematic errors have been accounted for. These errors are due to a multitude of small factors which change or fluctuate from one measurement to another and are due surely to chance.

\subsection{Errors in Transmitting Analog Signals}

\subsubsection{Digital Vs Analog signal}

Analog sensors generate analog signals. Digital Cameras generate digital signals. Today, nearly all electronic devices use digital. The main reason for the change from analog to digital is because digital signals are easier to transmit and are also more reliable. Plain and simple, a signal is the transmission of data.

\subsubsection{Analog Waveforms}

Analog signals were first used in the 1800's. They were used in conjunction with copper telephone lines to transmit conversations. These involved using two conductors for each line (send and receive). As technology progressed, an increasing number of people started using the telephone making analog signals too expensive and troublesome to maintain. Analog signals have picked up "noise" during its travel. Noise is simply an unwanted electrical or electromagnetic energy that degrades the quality of a signal. When the signal level crosses over the limits and has now become degraded and hard for the device on the receiving to interpret. Noise is sometimes called "distortion" or "clipping".

As signals travels across a wire, certain factor will add more "noise" to the signal. These factors can include air conditioning units, fluorescent lights, magnetic fields, etc. There are methods of separating or "filtering" noise from analog signals. However, most of these methods are not accurate, or are devices that transform the signals from analog to digital and back to analog. For these reasons, the use of digital signaling is used to provide a better delivery method.

\section{HARDWARE SETUP}

The hardware setup consists of a Intel core 2 duo CPU based industrial computer for operator station, Dedicated individual PC for image capturing unit, Color video cameras, Programmable logic controllers [3] and Ethernet switches.

\subsection{Structure of Control System}

In order to implement the remote control and monitoring system the general hardware and software configuration is established based on the application requirement. From the 
hardware point of view, programmable logic controller based control hardware's are used. The programmable Logic Controller is equipped with multiple I/O modules and a noninterruptible power supply for developing the control program. Program development system is used for developing the control program [7]. The resulting program is of type "ladder login" and was developed using an IBM -PC compatible computer and then downloaded into the PLC.

Digital industrial control systems can be either process based or discrete based. Process based controls [3] are used to control a continuous process of discrete based controls (otherwise known as batch controls) control discrete parts manufacturing or "batches" of material in a chemical plant. Both utilize the same types of control systems, sensors and networks. The Key control components of an industrial control system, including the control loop, the human machine interface through operator station and remote diagnostics and maintenance utilities. A control loop consists of sensors for measurement, control hardware, process actuators and communication of measurement variables.

\subsection{Major Control Components}

There are two system level supervisory based control schemes such as Distributed Control Systems (DCS) and Supervisory Control and Data Acquisition Systems (SCADA). DCS are used to control large, complex processes such as power plants, refineries and chemical plants typically at a single site. SCADA are used to control more dispersed assets where centralized data acquisition is as important as control. The DCS and SCADA components of a plant system typically reside on a peer to peer network.

\subsection{Control Station}

The heart if the control station is a standard Intel Pentium IV computer. The purpose of the computers is to execute the control and monitoring programs and image updating functions. Mat lab based interface software allows real time display [7] of various field images in accordance with the control operation. The program reads the live.dat file in server in sharable mode. The sharable file mode read operation is required because the PLC updates the same live.dat file in every $0.5 \mathrm{sec}$. Hence simultaneous access is allowed only in sharable mode. Field switched images are progressively reconstructed for final high-resolution field images and are displayed in the control Station.

\subsection{Image Capturing Unit}

The image capturing unit consists of an image capturing card with color image acquisition front end, dual ported color image buffer memory and a DSP for executing required image algorithms. It communicates controls and parameter information with the server. Instantaneous images are streamed for image transmitted.

\subsection{Programmable Logic Control}

PLCs are used for industrial control [3] today. A PLC has three basic functions, Control, Input and Output. Based on input and logic writer in the control (known as Ladder Logic, sequence or statement list), output are activated.

\section{SOFTWARE SETUP}

The Software setup consists of a PC-based user-interface component for progressive image transmission termed smartision screen copy, Command and Auto sequence programs termed PLC programs[13]and control and monitoring based image-processing [4] component termed Mimic. These components communicate through an Ethernet protocol. Following is a description of each component.

\subsection{PC based User-interface Component for Progressive Image Component}

It is responsible for progressively transmitting images from the video capturing unit. This is accomplished by first loading the image processing component onto the image capturing card. This component is specified in the settings. Second, the user-interface is presented. Third, images are transmitted to the control \& monitoring system. PC based user-interface component for progressive image transmission is a tool used to create auto screenshots in a very easy and quick way. With Auto screenshot, screenshot interval is selected. JPEG images [8] are files used to have better quality. The resulting executable file is "C: \ Program Files \ Screen Copy । SSC.exe"

\subsection{Auto Sequence Program}

This is responsible for executing the integration of image processing [4] with control and monitoring system in real time [2]. It achieves this by first communicating with the PC based component through Ethernet protocol for updated userinterface parameter and control information, second processing the image frame according to the image control panel requirements and third storing the control events for offline analysis.

\subsection{MATLAB Program}

Throughout this paper, MATLAB was used extensively as a test-bed for the development of the image processing based control and monitoring system. The result is a GUI based MATLAB tool termed AV for Active Vision image analysis. Reference to this application is made throughout the contents of this paper.

\section{TRANSPORT PROTOCOL}

Transmission Control Protocol (TCP or TCP/IP) and [15] User Datagram Protocol (UDP or UDP/ IP) are both transport protocols. These two major types of communication protocols are compared below.

\subsection{TCP versus UDP}

TCP provides connection-oriented communication (virtual circuit connection, like telephone communication). It manages a point-to-point and full-duplex connection for an application between two computers. It detects loss of messages and retransmits them. This TCP has efficient Congestion Control and flow control. It creates a connection before communication. TCP sends and receives data over this connection. Application data is considered to be a continuous unstructured stream of bits. Application programs must understand stream contents and boundaries of records. It closes a connection after communication. TCP guarantees reliable data delivery. The TCP recipient will receive data in a correct order without data loss or error.TCP allows multiple application programs on a given machine to communicate with programs in other machines.

UDP is another popular transport protocol. UDP is a Connectionless transport protocol. It does not acknowledge the packets. It does not detect of loss of messages nor retransmits them. It Ensures that data is received in the same 
order that it was sent. It has no congestion control and flow control. A UDP application sends messages without establishing and then closing a connection.UDP has a smaller overhead then TCP, especially when the total size of the messages is small.UDP does not guarantee reliable data delivery.UDP messages can be lost or duplicated, or they may arrive out of order; and they can arrive faster than the receiver can process them. The application Programmer using UDP has to consider and tackle these issues themselves.

\section{PROGRESSIVE IMAGE TRANSMISSION}

Real time field image has been important media for remote monitoring of field process. Initially video was captured and transmitted in analog form. The advent of digital integrated circuits and computers led to the digitization of video, and digital video enabled a revolution in the compression and communication of video. The growth and popularity of the network motivated video communication over best-effort packet networks. Video over best-effort packet networks is complicated by a number of factors including unknown and time-varying bandwidth, delay, and losses, as well as many additional issues such as how to fairly share the network resources amongst many flows and how to efficiently perform one-to-many communication for popular content.

A. Point-to-Point, Multicast Transmission

The common form of communication is point-to-point or oneto-one Communication. In point-to-point communications, an important property is whether or not there is a back channel between the receiver and sender. Another form of communication with properties that lie between point-to-point and broadcast is multicast. Multicast is a one-to-many communication. Field Images are captured and encoded for real-time monitoring.

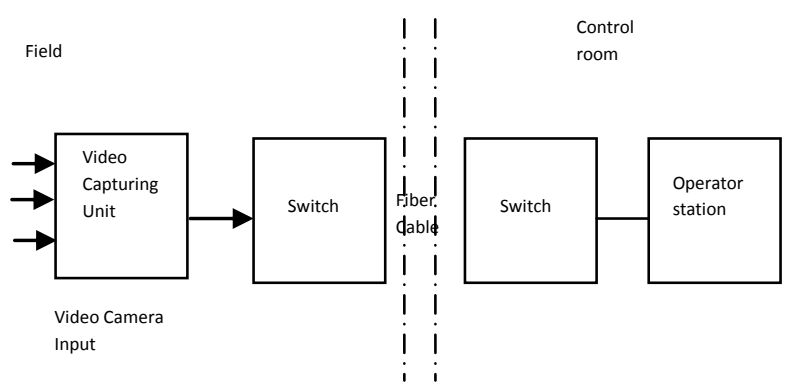

Fig 1: Video Encoder Diagram

\section{IMAGE COMPRESSION}

Uncompressed multimedia data (graphics, audio and video) data requires considerable storage capacity and transmission bandwidth. Despite rapid progress in mass storage density, processor speeds and digital communication system performance demand for data storage capacity and data transmission bandwidth continues to outperform the capabilities of available technologies. The recent growth of data intensive multimedia based web applications have not only sustained the need for more efficient ways to encode signals and images but have made compression of such signals central to storage and communication technology. Image compression [6] is achieved by exploiting the similarities or redundancies that exists in a typical video signal. For example, consecutive frames in a video sequence exhibit temporal redundancy since they typically contain the same objects, perhaps undergoing some movement between frames. Within a single frame there is spatial redundancy as the amplitudes of nearby pixels are often correlated. Similarly, the Red, Green, and Blue color components of given pixel are often correlated. Another goal of video compression is to reduce the irrelevancy in the video signal that is to only code video features that are perceptually important and not to waste valuable bits on information that is not perceptually important or irrelevant. Identifying and reducing the redundancy in a video signal is relatively straightforward, however identifying what is perceptually relevant and what is not is very difficult and therefore irrelevancy is difficult to exploit.

To begin with image compression is considered, such as the JPEG standard coders[5] generally degrade at low bit rates mainly because of the underlying block based discrete cosine transform. The following describes the JPEG compression process. JPEG divides up the image into 8 by 8 pixel blocks, and then calculates the discrete cosine transform (DCT) of each block. A quantizer rounds off the DCT coefficients according to the quantization matrix (can be image dependent). This step produces the "lossy" nature of JPEG, but allows for large compression ratios. JPEG's compression technique uses a variable length code such as run length codes or LZW on these coefficients. For decompression, JPEG recovers the quantized DCT coefficients from the compressed data stream, takes the inverse transform and displays the image. Basic idea behind this scheme is that the discrete cosine transform provides good energy compaction properties and hence we would require less number of bits to quantize and store these coefficients. Further, the quantized AC coefficients are mostly zeros and therefore can be run-length coded to give better compression ratios.

In a video sequence, it consists of a sequence of video frames or images. Each frame may be coded as a separate image, for example by independently applying JPEG like coding to each frame. However, since neighboring video frames are typically very similar much higher compression can be achieved by exploiting the similarity between frames. Currently, the most effective approach to exploit the similarity between frames is by coding a given frame [7] by first predicting it is based on a previously coded frame, and then coding the error in this prediction. Consecutive video frames typically contain the same imagery, however, possibly at different spatial locations because of motion. Therefore, to improve the predictability it is important to estimate the motion between the frames and then to form an appropriate prediction that compensates for the motion.

In order to achieve the lossless image compression [8] binary wavelet transform is used. An efficient architecture for lifting based 2-D discrete wavelet transform [9] is also used to compress the JPEG images. Recently the lossless image compression [10] is achieved by FELICS (Fast Efficient Lossless Image Compression) algorithm.

\section{PROGRESSIVE TRANSMISSION INTERFACE}

IMAGE

The main task of PIT is to encode the original image into a code stream. Image can be reconstructed effectively and efficiently by part of the code stream so that it can be transmitted in a progressive way. Bits with more importance should appear earlier in the code stream, so that users will always get the most important information in time and it will provide the best experience in viewing the transmitted image. While the first goal can be achieved easily by PIT techniques. 
Popular image standards such as JPEG and JPEG 2000 do support ROI coding. An attention model is used to extract the ROIs within the image. Attention model is given and it is used to extract the regions of interest. This attention mode has been successfully used in image adaptation, video summarization, and mobile picture browsing.

\section{RESULT AND DISCUSSION}

\subsection{Measurement of Liquid Level Parameter}

\subsubsection{Distance Matrix Algorithm}

By using the distance matrix algorithm, the current image is compared with standard image which is stored in the computer. When $\delta$ has the positive value, control signal is generated and it is given to the PLC for further action. When, $\delta$ has a zero value, control signal is not generated.

$$
\begin{aligned}
\delta=\sum_{i=1}^{n}\left(Y_{i}-F_{i}\right)^{2} & \\
\text { Where } \quad & =\text { Difference, } \\
\mathrm{Y}_{\mathrm{i}} & =\text { Standard Image, } \\
\mathrm{F}_{\mathrm{i}} & =\text { Field Image. }
\end{aligned}
$$

Test results are obtained by conducting experiments in the lab. A tank containing different liquid levels are photographed (through digital camera) for high level and low level. These are the STD images stored inside the computer (as shown in figure) Then the running level of liquid in the tank (during fill up) are photographed through digital camera in every two seconds and forwarded to the computer for comparison with the std image. When the current level image matches with the STD image control signal is generated. This signal is forwarded to the PLC for PID control. Figure 2 shows the test environment.

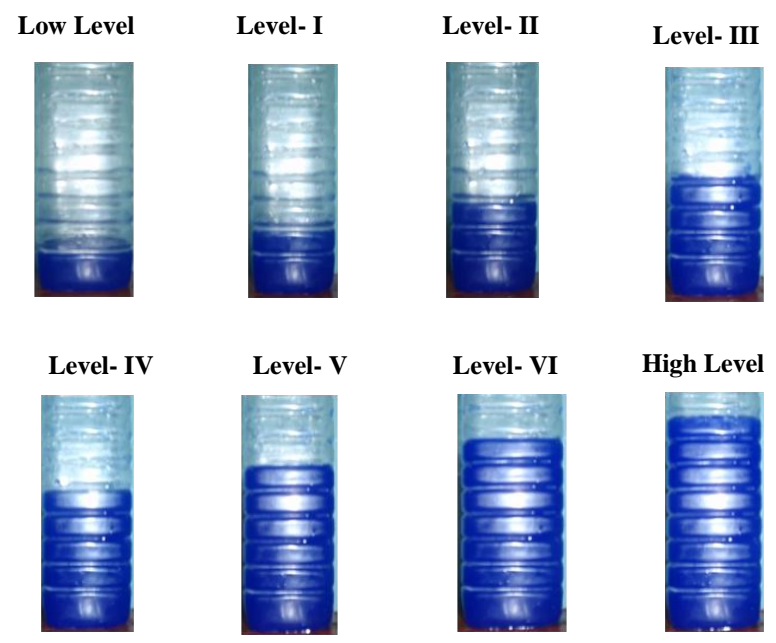

Fig 2 Test Environment to measure the liquid level

In fact only the concept is explained here. This level measurement can be utilized in high temperature boiler drum water level measurement in thermal power station where ordinary small sensor node usage is not possible. In Steel industries the steel level measurement is being done by the same procedure explained above. In coal burning furnaces in industries (Cement factory, process industry) coal powder and oil are mixed to burn and generate heat. Here proper level is to be maintained.

\subsubsection{Test to Verify the Liquid Level}

The testing purpose was to verify the liquid level measurement calculated by software with true measurement. In this testing the liquid level measurement testing was conducted with the following set up. First the liquid level calibration is done. $[11,12]$. The purpose of calibration is to find the linear scale of pixel to liquid level. The camera is placed $100 \mathrm{~cm}$ away from the tank as shown in figure 2 . With this set up, the distance of the laser spot to the image centre was 24 pixels. It meant that one pixel represents $24 / 100 \mathrm{~cm}$. Figure 3 indicates Testing set up to find out the liquid Level.

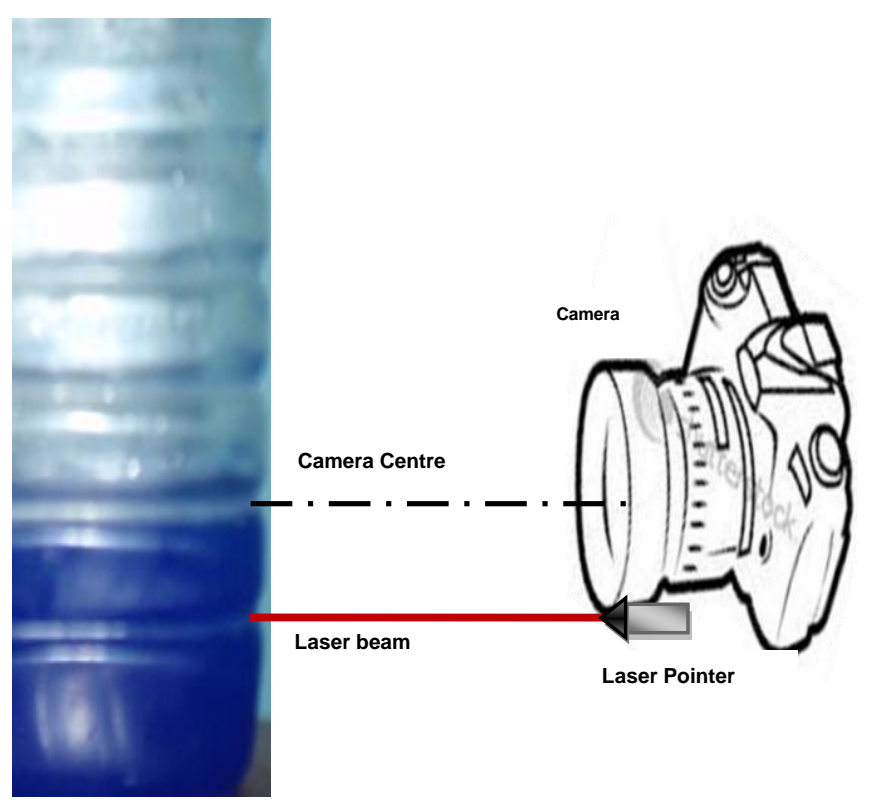

Fig 3 Testing setup to find out the liquid level

Table 1 True Liquid Level, Measured Liquid Level and its Error

\begin{tabular}{|c|c|c|c|c|}
\hline $\begin{array}{c}\text { SI. } \\
\text { No }\end{array}$ & $\begin{array}{c}\text { Liquid } \\
\text { Level }\end{array}$ & $\begin{array}{c}\text { Real } \\
\text { Liquid } \\
\text { Level(cm) }\end{array}$ & $\begin{array}{c}\text { Measured } \\
\text { Liquid } \\
\text { Level(cm) }\end{array}$ & \% Error \\
\hline 1 & $\begin{array}{c}\text { Low } \\
\text { Level }\end{array}$ & 2.5 & 2.4 & 4 \\
\hline 2 & Level -I & 4.0 & 4.08 & -2 \\
\hline 3 & Level -II & 5.5 & 5.58 & -1.45455 \\
\hline 4 & Level -III & 7.0 & 6.96 & 0.571429 \\
\hline 5 & Level -IV & 8.5 & 8.6 & -1.17647 \\
\hline 6 & Level-V & 10.0 & 10.04 & -0.4 \\
\hline 7 & Level-VI & 11.5 & 11.52 & -0.17391 \\
\hline 8 & $\begin{array}{c}\text { High } \\
\text { Level }\end{array}$ & 13.0 & 12.90 & 0.769231 \\
\hline \multicolumn{4}{|c|}{ Mean Absolute Error } & 0.13573 \\
\hline
\end{tabular}




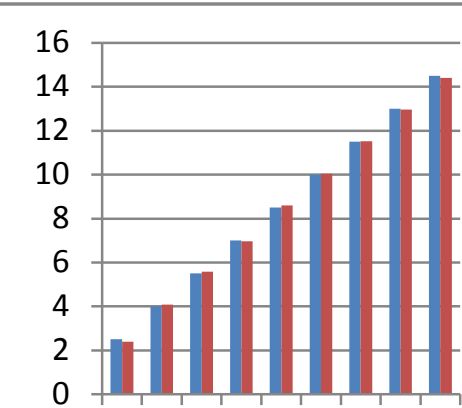

$\begin{array}{lllllllll}1 & 2 & 3 & 4 & 5 & 6 & 7 & 8 & 9\end{array}$

\section{Full Dry (Std Image)}

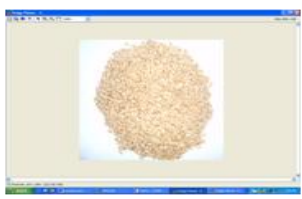

Partially Dry - 1

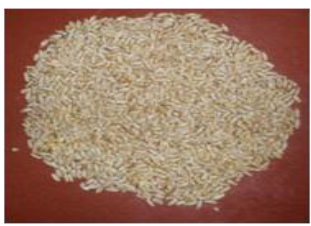

Partially Dry - 3

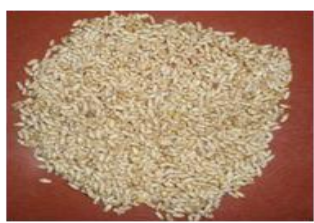

Full Wet (Std Image)

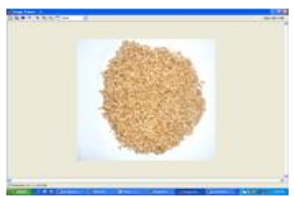

Partially Dry - 2

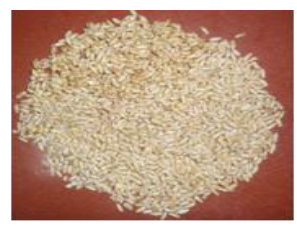

Partially Dry - 4

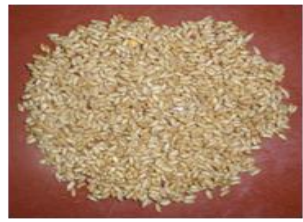

Fig 5 Test setup to measure moisture people during famine.

At present we are following the method of generating analog signal to find out the moisture content in the food grains of the storage by inserting a cylindrical capacitor in the storage. The capacitor produces different analog voltage signal based upon the moisture content. In fact the dielectric constant between the plates of the capacitor varies according to the moisture content to produce different permittivity value.

In present digital world we have state of the art technology namely Digital Image Processing (using software algorithms) which is replacing all the analog methods. We are also intending to replace the old method of using analog sensors to measure the moisture. Different photographs (digital camera image) are taken with different levels of moisture content of the food grains. These different moisture levels in the food grain photographs are stored inside the computer and database is created with the experimental setup values.

In the actual environment in the food storage unit, digital images are taken every one hour using digital camera (web camera) and forwarded to the PC. These images are compared with the already stored images of different moisture levels in the PC using suitable algorithm. When a particular incoming image matches with any one of the stored images, a control signal is generated to dry the food grains through a blower. The time of blower to dry the food grains varies according to the moisture level decided by the images. The Test set up is shown below: 


\subsection{Measurement of Speed}

In power station electricity is generated by coupling the turbine with the generator. The turbine is rotated through steam/water in thermal power station /Hydraulic Power station. In atomic power station heat is generated through nuclear fusion and water is heated and steam is generated to run the turbine. It is known that

$$
\mathrm{N}=\mathrm{F} * \mathrm{P} / 120
$$

$\mathrm{N}$ - Speed of turbine which is ultimately linked to generator $\mathrm{F}$ - Frequency

$\mathrm{P}$ - Number of poles in the Generator

\section{Current Image 1}

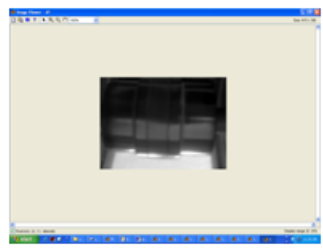

Current Image 2

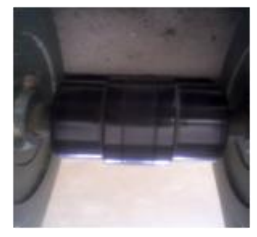

Current Image 4

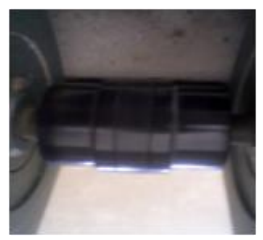

Fig 6 Various current images and Standard Images

It is essential to maintain constant speed $\mathrm{N}$ to maintain the frequency F. Increase/Decrease of frequency affect the domestic set up. To maintain constant speed the DIP Technique is suggested here. The coupling between the turbine and Generator is photographed at $5 \mathrm{sec}$ interval for rated constant speed and stored inside the computer as standard image. When the speed of the turbine varies due to less/ excess steam/water which ultimately affect the generator speed, a web camera which is installed near the coupling takes continuous photograph and forward the images to the computer. When the current image does not match the standard image control signal is generated and control action is taken accordingly. Figure 6 shows the various current images and Standard Images.

Table 3 demonstrates the value of $\delta$ after applying the image comparison algorithm and the corresponding actions to be taken place when it has positive value.
Table 3 The value of $\delta$ for speed measurement

\begin{tabular}{|c|c|c|c|c|}
\hline $\begin{array}{l}\text { Sl. } \\
\text { No }\end{array}$ & $\begin{array}{c}\delta \text { Value } \\
\text { using Image } \\
\text { Comparison } \\
\text { Algorithm }\end{array}$ & $\begin{array}{c}\text { Comparison } \\
\text { Among the } \\
\text { Images }\end{array}$ & Result & $\begin{array}{l}\text { Action to } \\
\text { be taken }\end{array}$ \\
\hline 1 & 0 & $\begin{array}{l}\text { Standard } \\
\text { Image } \\
\text { \& Current } \\
\text { Image } 1\end{array}$ & $\begin{array}{c}\text { Generator } \\
\text { is running } \\
\text { at a } \\
\text { constant } \\
\text { Speed }\end{array}$ & $\begin{array}{c}\text { No Control } \\
\text { Signal }\end{array}$ \\
\hline 2 & 0.1010 & $\begin{array}{l}\text { Standard } \\
\text { Image } \\
\text { \& Current } \\
\text { image } 2\end{array}$ & $\begin{array}{l}\text { Generator } \\
\text { is running } \\
\text { at a speed } \\
\text { less than } \\
\text { constant } \\
\text { Speed }\end{array}$ & $\begin{array}{l}\text { Control } \\
\text { Signal is } \\
\text { generated } \\
\text { and is given } \\
\text { to PLC for } \\
\text { further } \\
\text { Action }\end{array}$ \\
\hline 3 & 0.1012 & $\begin{array}{l}\text { Standard } \\
\text { Image } \\
\text { \& Current } \\
\text { image } 3\end{array}$ & $\begin{array}{l}\text { Generator } \\
\text { is not } \\
\text { running at } \\
\text { a constant } \\
\text { Speed }\end{array}$ & $\begin{array}{l}\text { Control } \\
\text { Signal is } \\
\text { generated } \\
\text { and is given } \\
\text { to PLC for } \\
\text { further } \\
\text { Action }\end{array}$ \\
\hline 4 & 0.09892 & $\begin{array}{l}\text { Standard } \\
\text { Image } \\
\text { \& Current } \\
\text { image } 4\end{array}$ & $\begin{array}{l}\text { Generator } \\
\text { is running } \\
\text { at a speed } \\
\text { less than a } \\
\text { rated speed }\end{array}$ & $\begin{array}{l}\text { Control } \\
\text { Signal is } \\
\text { generated } \\
\text { and is given } \\
\text { to PLC for } \\
\text { further } \\
\text { Action }\end{array}$ \\
\hline
\end{tabular}

\section{CONCLUSION}

The new interesting solution of Digital Image processing based online control and monitoring with the high level deterministic real-time capability seem to be very attractive for most demanding remote control applications. The novel architecture and methodology proposed for real-time control and monitoring with a rational network open - exciting possibilities in testing and performance analysis. Network with PLC endows control reliability to the system even under extreme conditions. The following table shows the performance of proposed method with existing methods with respect to various parameters.

Table 4 Performance of proposed method

\begin{tabular}{|c|c|c|c|c|}
\hline $\begin{array}{l}\text { Sl } \\
\text { No }\end{array}$ & Parameters & $\begin{array}{l}\text { Previous } \\
\text { Method }\end{array}$ & $\begin{array}{l}\text { Present } \\
\text { Method }\end{array}$ & $\begin{array}{c}\text { Proposed } \\
\text { method }\end{array}$ \\
\hline 1 & $\begin{array}{l}\text { Responsive } \\
\text { Time }\end{array}$ & $5 \mathrm{sec}$ & $45 \mathrm{~ms}$ & $15 \mathrm{~ms}$ \\
\hline 2 & Accuracy & $80 \%$ & $85 \%$ & $98 \%$ \\
\hline 3 & Calibration & $\begin{array}{l}\text { Twice a } \\
\text { Year }\end{array}$ & $\begin{array}{l}\text { Twice a } \\
\text { Year }\end{array}$ & $\begin{array}{c}\text { Not } \\
\text { Required }\end{array}$ \\
\hline 4 & Hysteresis & Normal & Normal & NA \\
\hline 5 & Operation & Manual & $\begin{array}{c}\text { Partially } \\
\text { Automated }\end{array}$ & $\begin{array}{c}\text { Fully } \\
\text { Automated }\end{array}$ \\
\hline 7 & $\begin{array}{l}\text { Quality Of } \\
\text { Signal } \\
\text { Transmission }\end{array}$ & $\begin{array}{l}\text { Max } \\
\text { error }\end{array}$ & $\begin{array}{l}\text { Normal } \\
\text { Error }\end{array}$ & $\begin{array}{l}\text { Minimum } \\
\text { Error }\end{array}$ \\
\hline 8 & $\begin{array}{c}\text { Mean Absolute } \\
\text { Error }\end{array}$ & $7.4 \%$ & $5.2 \%$ & $0.14 \%$ \\
\hline
\end{tabular}


The new interesting solution by concept of integrating real time field images by removing separate TVs will lead to a Digital Factory. This digital factory will perform the complete removal of analog errors which are occurred when analog signal sensors are used to measure various physical parameters in industry .This novel architecture opens exciting possibilities in testing fault diagnosis, real time control, performance analysis and drastic improvement on production capacity. Here the new interesting solution of concept of integrating real time field images by removing separate TVs using the high level deterministic real-time capability seem to be very attractive for most demanding remote control applications. The novel architecture and methodology is proposed for real-time control and monitoring with a rational network open exciting possibilities in testing and performance analysis. Network with PLC endows control reliability to the system even under extreme conditions.

\section{REFERENCES}

[1] C.S. Kim, R.C.Kim "Robust transmission of video sequence using double vector compensation," IEEE, Circuit Vol. 11, PP 1011-1021, Sept 2001.

[2] Halang W.A., Stoyenko A.D (2004). "Constructing Predictable Real-Time Systems". Kluwer Academic Publishers,.

[3] Parr, E.A. (1995), Programmable Controllers, An Engineer's Guide Newnes, an imprint of Butterworth Heinemann Ltd. Oxford, pp325.

[4] L. Chiariglione, "The development of an integrated audiovisual coding standard: MPEG," Proc. IEEE, vol.83, pp. 151-157, Feb 2004.

[5] Athanasios skodras "The JPEG 2000 still image compression standard" IEEE, Signal processing, Sept 2001.

[6] Athanasios Leontaris "Video compression for lossy packet networks with mode switching and dual frame buffer," IEEE, Image processing vol.13, No 7, July 2004.

[7] L. Chiariglione, "The development of an integrated audiovisual coding standard: MPEG," Proc.IEEE, vol.83, pp.151-157, Feb 2004.

[8] H.Pan,W.C.Siu and N.F.Law "Lossless image Compression Using Binary Wavelet Transform" IET Image Process 2007, I,(4) pp 353-362

[9] Peng Cao,Xin Guo, Chao Wang and Jie Li "Efficient architecture for 2 D Discrete Wavelet Transform based on Lifting Scheme "1-4244-1132-7/07\$25.00@2007 IEEE

[10] Tsung-Han Tsai, Yu-Hsuan Lee, Yu-Yu Lee "Design and Analysis of High-Throughput loss less Image compression Engine using VLSI oriented FELICS Algorithm" IEEE Transactions on Very Large Scale
Integration(VLSI) Systems, 1063-8210/\$25.00 (C) 2009 IEEE.

[11] Muljowidodo.K.,Mochammed.A.Rasid,Saptoadi.N.,Agus Budiyono "Vision Based distance Measurement System Using single Laser Pointer design for underwater Vehicle" Indian Journal of Marine Sciences,Vol.38(3),Sep 2009,PP 324-331.

[12] Ti-Ho Wang,Ming-Chih lu,Wei Yen Wang,Cheng Yen Tsai “ Distance Mesurement using single non metric CCD camera",Proceedings of $7^{\mathrm{th}}$ conf on Signal Processing Computational Geometry \& Artificial Vision,Atens, Greece,Aug,2007.

[13] K. Rajappan 'A.Justin Diraviam ,R.S.D.Wahida Banu, “ Digital Image Processing Based on line Monitoring And Control" Inter nation Journal of Image Processing and Networking Techniques,Vol.2.Issue 1,page 67-77.

[14] Andrew Secker and David Taubman, " Highly Scalable video Compression with Scalable motion coding" IEEE Image Processing,Vol13,No.8 , August. 2004.

[15] H.Schulzrine,A.Rao “ Real Time Streaming Protocol” IETF,RFC 2326,1998.

\section{AUTHORS PROFILE}

Justin Diraviam received the B.E(EEE) from M.S university ,M.E(Applied electronics) from M.K university, Tamilnadu, India.. Currently he is working as Asst. Prof in CSE dept in Sardar Raja College of Engineering, Tirunelveli, Tamilnadu. He has published several papers in national and international conferences. His areas of interest are Digital image processing, neural network and low power VLSI design.

S.P.Victor earned his M.C.A degree from Bharathidasan University, Tiruchirapalli and Ph.D. degree in computer Science from M.S. University, Tirunelveli. His Ph.D. thesis was on parallel algorithms. He is currently working as Reader in the department of Computer science, St.Xavier's College (Autonomous), Palayamkottai, Tirunelveli. He has published research papers on parallel algorithms in International, National journals and conference proceedings. He was organizing secretary of the National Conference on Emerging Trends in Algorithms held at Tirunelveli in March 2006.

K.Rajappan received the B.E (ECE) from Madras University, M.Tech in electronics from Rajasthan University and $\mathrm{Ph} . \mathrm{D}$ from international university in U.S.A. Currently he is working as principal in Sri Subramanya College of Engineering and technology, Palani, Tamilnadu. He has published several papers in national and international conferences. His areas of interest are image processing and neural network. 